\title{
Out of Adversity comes Innovation, How is Saudi Arabia Achieving a Low Case Fatality Rate during the Coronavirus Pandemic?
}

\author{
Anhar Hamza* \\ Department of Medical Protocol, King Abdulaziz Medical City-Riyadh, Ministry of National Guard Health Affairs, King Abdullah International Medical \\ Research Center (KAIMRC), King Saud bin Abdulaziz University for Health (KSAU), Saudi Arabia
}

*Corresponding author: Anhar Hamza, Medical Protocol Department King Abdulaziz Medical City-Riyadh, National Guard Health Affairs, Mail code 1407, PO Box. 22490 Riyadh, 11426, Kingdom of Saudi Arabia.

To Cite This Article: Anhar Hamza. Out of Adversity comes Innovation, How is Saudi Arabia Achieving a Low Case Fatality Rate during the Coronavirus Pandemic?. 2020 - 9(2). AJBSR.MS.ID.001365. DOI: 10.34297/AJBSR.2020.09.001365.

Received: 䟧 May 13, 2020; Published: 㘹 June 12, 2020

\begin{abstract}
In the Coronavirus pandemic, desperate times called for desperate measures and this is how Saudi Arabia maintained a low case fatality rate. Early public awareness programs and border closure led to a reduction in the rate of infection especially in those at high risk of mortality from the disease. At a clinical level, adoption of virtual clinics for routine outpatient care allowed the country to focus its efforts on the provision of COVID-19 care in specially adapted isolation centers. On a psychological level, the health care providers were well looked after which led to the provision of effective patient care.
\end{abstract}

Keywords: COVID-19; Saudi Arabia; Frontline staff; MNGHA; Pandemic; Case fatality rate (CFR)

\section{Introduction}

It has now been more than four months since the first case of the Novel Corona Virus 19 (COVID-19) was first reported in Wuhan in China's Hubei province [1]. Unfortunately, as we stand today (7th of May 2020), more than 3.8 million people have been infected with more than 270 thousand deaths worldwide [2].

The World Health Organisation (WHO) recommended that each country should "suppress community transmission" through context-appropriate infection prevention and control measures, and appropriate and proportionate restrictions of non-essential domestic and international travel [3]. The WHO led the world in launching strategic preparedness and response plans that were adapted by each country. Some countries initially chose the principle of "heard immunity" in an attempt to gain passive population immunity and flatten the curve of infection, others chose to impose no restrictions and deal with the COVID infection as it unravels. Saudi Arabia, on the other hand, chose to instigate early lockdown of schools and educational institutions, 24-hour curfew, robust public awareness campaign, early screening and hospitalization of confirmed COVID-19 cases (Saudi Ministry of Health (MOH)). This was in massive contrast to the advice adopted by some countries of self-isolation of suspected and confirmed cases to avoid overwhelming the health system.

According to the WHO, the top five global public health and social measure interventions include travel restrictions, school closures, limiting public gatherings, introduction of quarantine policies and border closure.

\section{Discussion}

When reports of the COVID-19 were first published, scientists were lulling themselves into a false sense of security that this disease has a low case fatality rate (CFR) of 1.4\% [3] unlike the 9.6\% of the Severe Acute Respiratory Syndrome (SARS) or the 36\% of the Middle East Respiratory Syndrome (MERS) [4]. Despite the much higher CFRs for SARS and MERS, COVID-19 has led to more total deaths due to the large number of cases. 
As of the 7th of May 2020, we are now only 130 days on since this disease was first reported, and the WHO is estimating the current worldwide CFR to be over 3\% [3]. The average case fatality rate has therefore doubled in the interim. One has to bear in mind that the case fatality rate is actually much higher in certain countries such as China (5.5\%); where the pandemic has started, USA (6\%), Italy (13.9\%) and the United Kingdom (14.7\%) to name but a few (these figures are calculated from the John Hopkins University of Medicine Coronavirus Resource Center website [2]. The CFR figures vary depending on the number of people affected, the point at which the country is in at the trajectory of its outbreak and the rate of accessibility to testing. Interestingly however, Saudi Arabia is amongst the countries with the lowest fatality rate of $0.64 \%$ despite performing over 433 thousand tests (239 deaths out of 37'136 confirmed COVID cases). The question that begs itself here is, how did Saudi Arabia manage to achieve this low CFR?

On the 11th of March 2020 and as the number of COVID-19 cases outside China had increased significantly involving 114 countries and over 4'000 deaths, the WHO declared the COVID-19 a pandemic. At that time, Saudi Arabia had already stopped pilgrimage to the holy sites by the 5th of March, schools by the 9th of March and international boarders were subsequently closed by the 15th of March 2020 [5]. As such, Saudi Arabia was amongst the first countries that locked their borders banning international and local travel. Immediate paid holiday and self-isolation was offered to those coming from outside the kingdom. They were also thoughtful about the social distancing and curfew (also known as lockdown) rules where it was announced at a short notice. This was presumably planned to avoid some of the pitfalls other countries found themselves in when they announced lockdown dates in advance. Some of the pitfalls included panic buying and long queues at doctor's surgeries which may potentially have increased the risk of exposure to the COVID-19 virus. The curfew rules were also being monitored continuously as the pandemic unfolded and were relaxed when the number of new reported cases decreased to allow people to have near normality in between.

The Muslims within Saudi Arabia are currently observing the Holy month of Ramadan and the Eid celebrations that follow. It is the cultural norm that during these months, family and friends would gather and people would visit the Holy cities of Mecca and Madina. However, any form of travel was banned by the authorities including gatherings for prayers in the holy cities or within mosques. To further demonstrate the seriousness of these measures, penalties of over two and a half thousand dollars were introduced to people driving without a valid permit during curfew hours.

The Saudi MOH put huge emphasis on early prevention and public awareness campaigns. Educational mobile alerts were being sent about the subtle symptoms of COVID-19 and the simple measures to prevent transmission. All the mobile networks changed their logo to "stay home" and instead of a dialing tone, one would get a brief message to encourage people to stay at home and to remain safe.

Although outpatients and routine face-to-face clinics were suspended, elderly patients and those with chronic conditions were offered virtual clinics to maintain their continuity of care. "Your medication to your door" is an initiative to deliver prescriptions to patients at their homes. The patient would fill a medication request through the mobile application and the medication would arrive by dedicated courier companies provided by the government.

Most importantly perhaps, COVID-19 patients were quarantined regardless of their symptoms. Symptomatic patients were admitted to a dedicated isolation hospital whereas asymptomatic patients were kept in a dedicated isolation hotel for observation and monitoring. The public health team who list all the contacts and get in touch with them perform contact tracing. These proactive steps were fundamental in preventing further community spread, and in observing early signs of deterioration. An electronic COVID-19 reporting form was developed so that it is submitted directly to $\mathrm{MOH}$ [6]. This shows the appreciation of the need for transparency with the public and the importance of accurate data collection upon which strategic plans are adjusted accordingly.

In my view, the most successful strategy was looking after frontline staff and providing them with the necessary personal protective equipment (PPE). A large proportion of frontline staff were fit tested for the specialized face masks. They were also briefed about crisis plans where isolation hospitals and back up quarantines were nominated and prepared early on in the pandemic. During times where there was controversy as to whether healthcare providers should wear masks or not, the Ministry of National Guard Health Affairs (MNGHA) reinforced the implementation of universal masking by all healthcare workers all the time inside and outside the clinical area [7].

In MNGHA, the patient safety department were continuously monitoring the wellbeing of staff through anonymous surveys. The Mental Health team, under the auspices of the Chief Executive Officer, launched a "be well" online support network program for healthcare professionals. This was nicely married up with free online webinars on how to stay well during the pandemic. A variety of webinars were offered to allow practitioners to continue their continuous professional development and more importantly, to discuss issues such as managing stress and how to use the current pandemic as an opportunity to better oneself. The health sciences library produced a link that provides a guide to recent medical literature published about COVID19. 
As a healthcare provider, I was honored when the director of our protocol department attended in person to inform us that one of our patient-escort colleagues developed COVID-19 and that testing is offered to those who were in close contact and any concerned member of staff. Clinics were temporarily suspended until the entire staff test results were known. Another measure was put in place whereby staff were divided into two teams that do not physically meet at all. The two teams work on a Rota basis to ensure that if any member became infected, there was always a backup team to look after the patients.

A newly built simulation center was dedicated to screening frontline staff. It was chosen because of its proximity to the main site hospital and easy access to staff. It is well set up to accommodate symptomatic and asymptomatic staff in two separate areas. They had a dedicated infection control team responsible for filling the screening questionnaires and recording the vital signs. An infection control physician reviews you before having a nasopharyngeal swap taken. An advice sheet is provided, and you are advised to selfisolate until you receive a call from the infection control team with the swap results, which is often within 24-hours. If the swap results are negative, you continue to get a daily-automated call from the $\mathrm{MOH}$ asking you if you have developed any symptoms and directing you to their 24-hour COVID-19 helpline.

MNGHA launched a 24-hour call center dedicated to employees and their dependents to answer all questions and consultations related to COVID-19. A team consisting of Emergency Medicine consultants, infection control specialist and patient relations officers in addition to Emergency Medical Services and communication staff, are all liaising together to answer enquiries. This is to provide comfort to all MNGHA staff and their dependents, and to help them stay in their homes safely. An agreement was reached with some major hypermarkets to secure faster delivery of purchased grocery and personal items on daily basis for MNGHA staff.

\section{Conclusion}

By putting societies and economies on hold, Saudi Arabia has curtailed the ability of the virus to continue to affect more lives. One of the lessons that we have learnt thus far is that the faster all cases are found, tested and isolated, the harder we make it for COVID-19 virus to spread. Implementing the above measures meant that Saudi Arabia managed to a certain extent to meet the WHO's goal of controlling the pandemic by slowing down the transmission and reducing mortality associated with COVID-19.
All the prevention measures have translated into fewer intensive care admissions and consequently fewer fatalities. The simple anticipatory measures mentioned above meant that the health system was prepared to accommodate the severe cases and offer an efficient yet sustainable quality of care.

It is undoubtedly difficult balancing all the costly prevention and health provision measures against individual countries' economy. The sooner we end this pandemic, the sooner we can return to normal safe life, which will revive the economy. As such, by applying some of the measures mentioned above, the manpower and resources can be appropriately redirected towards the current pandemic. Reducing the infection rate and saving lives should be every country's top priority. Furthermore, looking after the healthcare provider is of paramount importance to ensuring safe and effective provision of healthcare.

\section{Conflict of Interest}

None reported.

\section{Funding/Support}

The author received no financial support for the research, authorship, and publication of this article.

Disclaimer: The opinions expressed herein reflect the views of the author and do not necessarily represent the official position of the Government of Saudi Arabia.

Acknowledgments: I would like to thank our CEO Dr Bandar Al Knawy and our department Head, Dr Fahad Alhemoudi for their continual guidance and support and for looking after their staff during this pandemic.

\section{References}

1. (2020) Pneumonia of unknown cause - China. Disease outbreak news World Health Organisation.

2. (2020) John Hopkins University of Medicine Coronavirus Resource Center.

3. (2020) Overcoming COVID-19. World Health Organisation.

4. Wu Z, McGoogan JM (2020) Characteristics of and important lessons from the coronavirus disease 2019 (COVID-19) outbreak in China: summary of a report of 72314 cases from the Chinese Center for Disease Control and Prevention. Jama 323(13): 1239-1242.

5. (2020) The World Health Organisation.

6. (2020) COVID 19 Dashboard: Saudi Arabia. Saudi Ministry of Health Website.

7. (2020) Ministry of Health. Saudi Arabia Ministry of National Guard Health Affairs, National Guard. 